\title{
Movimenti della faglia del Iago di Cavazzo, in relazione con la locale caduta di pioggia
}

\author{
P. Caloi * M. Migani ** \\ Ricevuto il 15 Ottobre 1971
}

\begin{abstract}
Riassunto. - Si portano chiare testimonianze sull'esistenza di nicromovimenti della faglia del Lago di Cavazzo ('Tolmezzo), associati alla locale caduta di pioggia. Essi vengono registrati, sotto forma di rotazioni della verticale, dalle stazioni clinografiche sistemate nella centrale "Enel " di Somplago, ricavata in roceia. La centrale si trova sul bordo occidentale della grande faglia del lagro. A sua volta la faglia del lago - che probabilmente si prolunga fino alla confluenza del torrente Melò nel Tagliamento, ca $10 \mathrm{~km}$ a Sud della Centrale - si trova nel quadrante NW della vasta zona di alluvioni del Tagliamento, che si stende fra Venzone, Gemona e Osoppo. La rotazione della verticale verso SSE può essere attribuita all'appesantimento della zona alluvionale, dovuto alla pioggia: la deviazione risulta infatti tanto maggiore quanto più cospicua è la caduta di pioggia. ì da ritenere che sotto l'esteso letto del fiume Tagliamento, esista una fiumana, alimentata dalla pioggia d'infiltrazione. A questo riguardo, è da rilevare che, durante i mesi estivi, quando l'apporto della pioggia diminuisce, la verticale subisce a Somplago un lento movimento in senso contrario, come per un corrispondente lento alleggerimento della zona a SSE.

I micromovimenti relativi dei due bordi della faglia possono avere un effetto "decongestionante "della zona da essa interessata, nel senso di impedire notevoli accumuli di tensioni elastiche nel mezzo circostante. Sta di fatto che, malgrado l'elevata sismiciti della Carnia, nei tempi storici la zona di Somplago non si è mai trovata all'epicentro di terremoti distruttivi.
\end{abstract}

1. - E stata data notizia del funzionamento - in fase sperimentale - di due pendoli orizzontali di eccezionale potenza (1). sistemati in un pozzo di 136 metri di altezza, ricavato nel sistema roccioso, in cui si trovano le attrezzature della Centrale " Enel " di Somplago (Tolmezzo).

* Direttore d'Osservatorio, Istituto Nazionale di Geofisica, Roma.

** E.N.EL. - Compartimento di Venezia. 
In condizioni normali, le registrazioni dei pendoli sono quelle associate alle maree terrestri. Son mancano perturbazioni locali, legate ai movimenti dell'acqua nel pozzo piezometrico della Centrale; comunque, facilmente distinguibili.

Frequentemente, si presentano perturbazioni anormali, di origine tettonica o attribuibili a cause esterne.

ì di queste ultime che intendiamo trattare in ciò che segue.

2. - Non costituisce certo una novita la registrazione di variazioni della verticale, associate al transito di più o meno accentuate variazioni della pressione atmosferica. Negli ultimi decenni, constatazioni del genere si sono moltiplicate.

Ci risulta invece del tutto nuova l'estrema sensibilità a questi, e ad altri fenomeni al essi collegati, quale si verifica presso la stazione clinografica di Somplago.

Da quando la stazione di pendoli orizzontali funziona presso la Centrale idroelettrica di Somplago, la casistica si è fatta così copiosa da togliere qualsiasi clubbio al riguardo; non c'è passaggio di perturbazione atmosferica di qualche intensità che non provochi la più o meno rapirla fuoruscita dell'immagine di una delle due componenti (la S33º dal foglio-registratore, causando sbandamenti accentuati anche nell'altra componente a $90^{\circ}$.

Ci limitiamo a riportare qualche esempio, dei più recenti: $20-25$ Febbraio 1969 (fig. 1), 21-26 Aprile 1969 (lig. 2), 16-20 Maggio 1969 (fig. 3), 3-9 Giugno 1969 (fig. 4), 7-13 Luglio 1969 (fig. 5) e numerosi altri.

Una prima visione delle curve clinografiche e della pressione atmosferica messe a confronto può far credere al una chiara rispondenza fra deviazione (più o meno accentuata) della componente clinografica N33ㅇ-S330 E la diminuzione della pressione atmosferica osservata nella zona. Una più attenta indagine mette però in risalto clue fatti inesplicabili: la difformità nei tempi d'insorgenza dei due eventi (variazione della verticale e climinuzione della pressione atmosferica) e apparente indipendenza dell'ampiezza secondo cui i due eventi si manifestano, nel senso che a piccole variazioni della pressione possono corrispondere forti deviazioni della verticale, o viceversa.

A questo punto, ci siamo posti una domancla: l'improvvisa, ampia deviazione della " $A$ " verso $\$ 33^{\circ} \mathrm{E}$, seguita da rapida fuoruscita dell'immagine dal registratore è davvero legata - ed in morlo esclusivo al passaggio di un minimo di pressione su Somplago? Un fatto è certo: generalmente, quando la pressione atmosferica subisce un forte balzo 
negativo, si verifica la deviazione della verticale che porta l'immagine luminosa fuori del registratore. Non è sempre vero però il contrario, nel senso che sovente si ha ampia fuoruscita dell'immagine verso S330 $\mathrm{A}$, senza che la pressione atmosferica abbia subíto variazioni fuori del normale. Ci siamo chiesti se ciò poteva essere attribuito ad eccezionali alte maree nelle lagune venete (Grado), ma la risposta fu negativa. Escluso che fenomeni del genere fossero provocati da variazioni di livello del vicino lago di Cavazzo - che giomalmente subisce diminuzioni od aumenti di qualche decimetro, conseguenti alle richieste della vicina centrale - (d'altronde, lago di superficie troppo limitata, perché variazioni d'invaso, anche notevoli, possano provocare effetti tanto vistosi), giudicato del tutto improbabile che un fenomeno cosi regolare e tanto frequente fosse legato ad un'origine tettonica, si pensò ad un'altra manifestazione meteorologica: la pioggia. E così è. Per citare un solo esempio, si consideri l'evento del 19 Novembre 1970. Come risulta dalla. fig. 9, la variazione della pressione atmosferica fu, in quel giorno, di trascurabile entità; ciò non pertanto, l'immagine della comp. " $A$ " del pendolo orizzontale andò rapidamente ed abbondantemente fuori campo. Ia carluta di pioggia di quel giorno - solo a Somplago, oltre $90 \mathrm{~mm}$ in $24^{\mathrm{h}}$ - giustifica la forte improvvisa variazione della verticale, verificatasi nella zona. E ricorrendo alla pioggia, si giustificano pure lutte le variazioni di verticale osservate, oltre a quelle riportate nelle figg. 1-16.

3. - Perchó una così acnta sensibilità a fenomeni che altrove passano pressoché inosservati? Non dimentichiamo che il pendolo ha una sospensione (quella superiore) che si stende verticalmente per ben $130 \mathrm{~m}$ nell'interno del monte. C'era un'azione differenziale fra il punto d'attacco superiore e quello inferiore, alla base della Centrale idroelettrica, capace di esaltare l'efietto di rotazione? O tutto il monte ruotava come un mezzo rigido? L'alternativa poteva essere sciolta con un pendolo orizzontale, condizionato esclusivamente dal suo supporto-base. ì quello che ì stato fatto. Il Dott. Migani ha provveduto alla costruzione e sistemazione di due pendoli orizzontali, sistemati sul pianobase della Centrale, nei pressi della stazione sismica e dell'altra stazione clinografica. I nuovi pendoli, essendo di sensibilità molto inferiore a quella della primitiva coppia, presentavano - nei confronti di quest'ultima - il vantaggio di consentire - generalmente - la registrazione completa del fenomeno, fatta eccezione per casi di variazioni della verticale particolarmente accentuati. 
Ise registrazioni della nuova coppia di pendoli hanno consentito di provare che, in effetti, è tutto il monte a subire uniformemente l'inclinazione, anche nel caso di perturbazioni superficiali, come quella in esame.

Le figg. 14-16 rappresentano esempi di registrazione del fenomeno, da parte della nuova coppia di pendoli orizzontali.

4. - Isa carluta di pioggia nella zona circostante Somplago è quindi la principale responsabile della deviazione della verticale, accusata dalle stazioni clinografiche, sistemate nella Centrale elettrica, ricavata in roccia all'estremo Nord del lago di Cavazzo.

Come spiegare tanta sensibilità delle stazioni clinografiche alla caduta di pioggia? Viene spontaneo pensare ad una particolarità tettonica della zona, dove le stazioni accennate hanno la loro sede.

I geologi osservano che il motivo tettonico dominante della regione ¿̀ dato da una serie di scaglie tettoniche, parallele e sovrascorse verso $\mathrm{S}$ e SW, mediante superficie di accavallamento, con immersione verso $\mathrm{N}$. Si hanno inoltre faglie trasversali. Una delle più importanti - scoperta e studiata per primo da E. Feruglio $\left({ }^{4}\right)$ - è la faglia riconoscibile lungo il fianco occidentale del lago di Cavazzo (figg. 18, 19). Trattasi di frattura dovuta a spostamento orizzontale, in senso trasverso alle pieghe, segnato dalla loro brusca deviazione verso $S$, in corrispondenza della valle del Melò. Il piano di frattura è accompagnato da rigetto verticale; intorno ad esso, la roccia ha subito un minuto stritolamento.

La frattura si può seguire solo per un breve tratto, in quanto sia a Nord che a Sud si nasconde sotto le acque del lago e le alluvioni. Non è possibile quindi accertare se essa prosegua - come ritiene R. Selli $\left(^{5}\right)$ fino allo sbocco del Melò nel Tagliamento, in prossimità del quale, sul lato Ovest del Nelo, appare netta un'altra frattura trasversa (fig. 18).

Tale faglia risale alle fasi orogenetiche più recenti (post-elveziane). Fra l'elveziano e il quaternario si ha la fase di diastrofismo più intenso: spinte unilaterali, provenienti da Sud, determinano un cospicuo costipamento, seguito da sollevamento delle pieghe montane e dal loro rovesciamento o scorrimento; mentre un'energica compressione da Est provoca la loro torsione, con formazione di fratture trasversali(*).

(*) A questo proposito, va messo in risalto il fatto che, a prescindere dal fenomeno oggetio di questo studio, da quando funziona la stazione clinografica di Somplago, è sempre presente una lenta deviazione verso Nord. Il permanere di una microrotazione verso Nord (generalmente verso $\mathrm{NW}$; talora verso NE), può derivare da una causa tettonica, come una persistente spinta da Sud nella zona. 


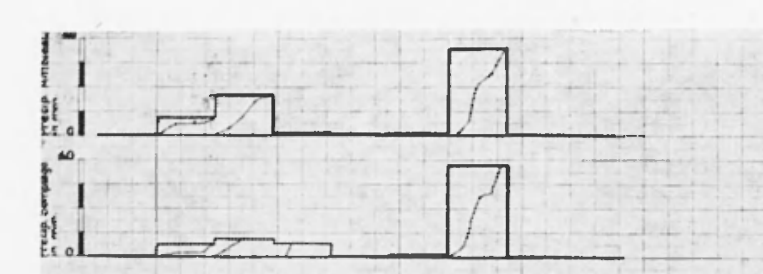

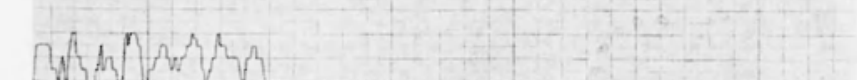
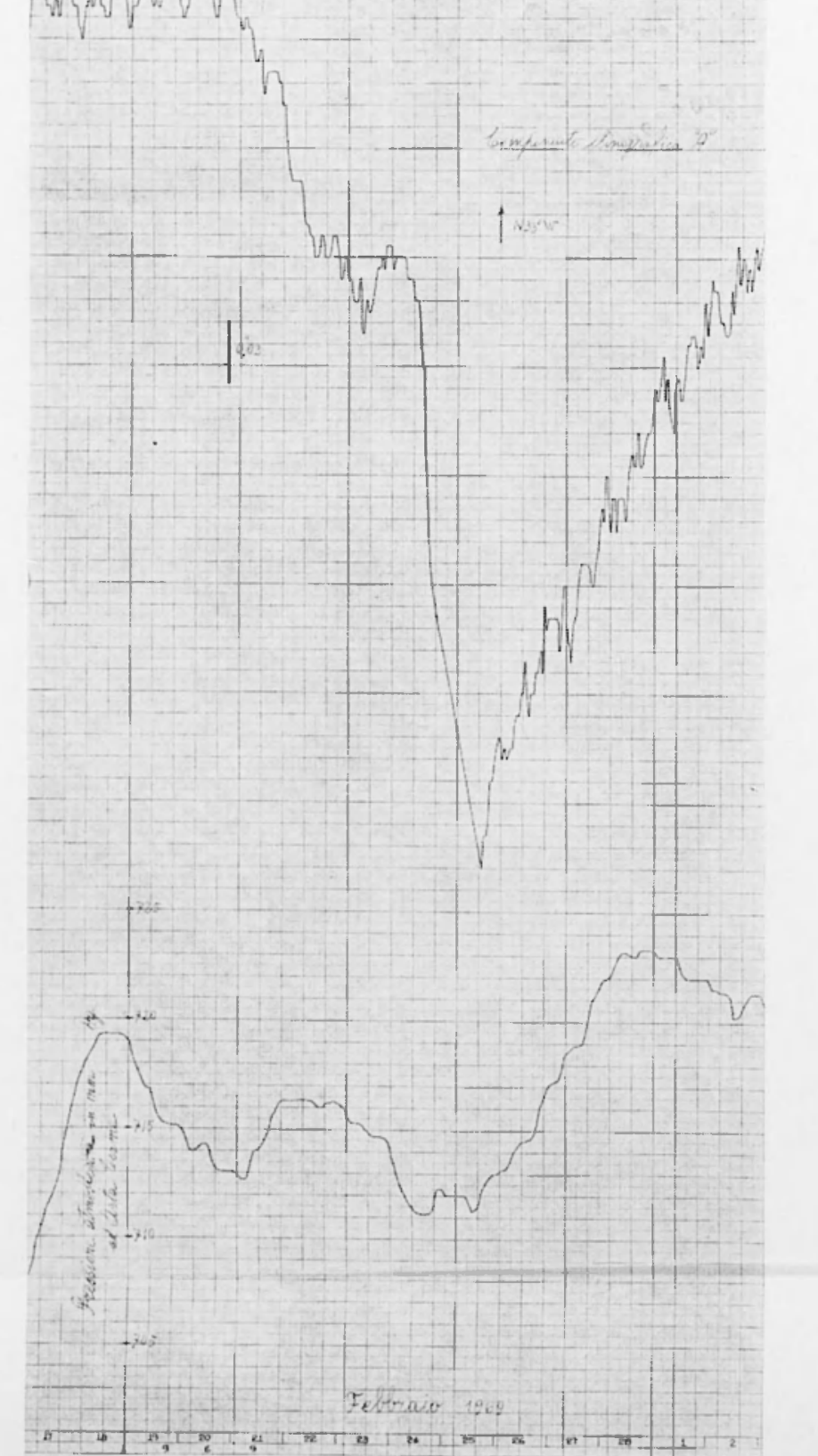

1.
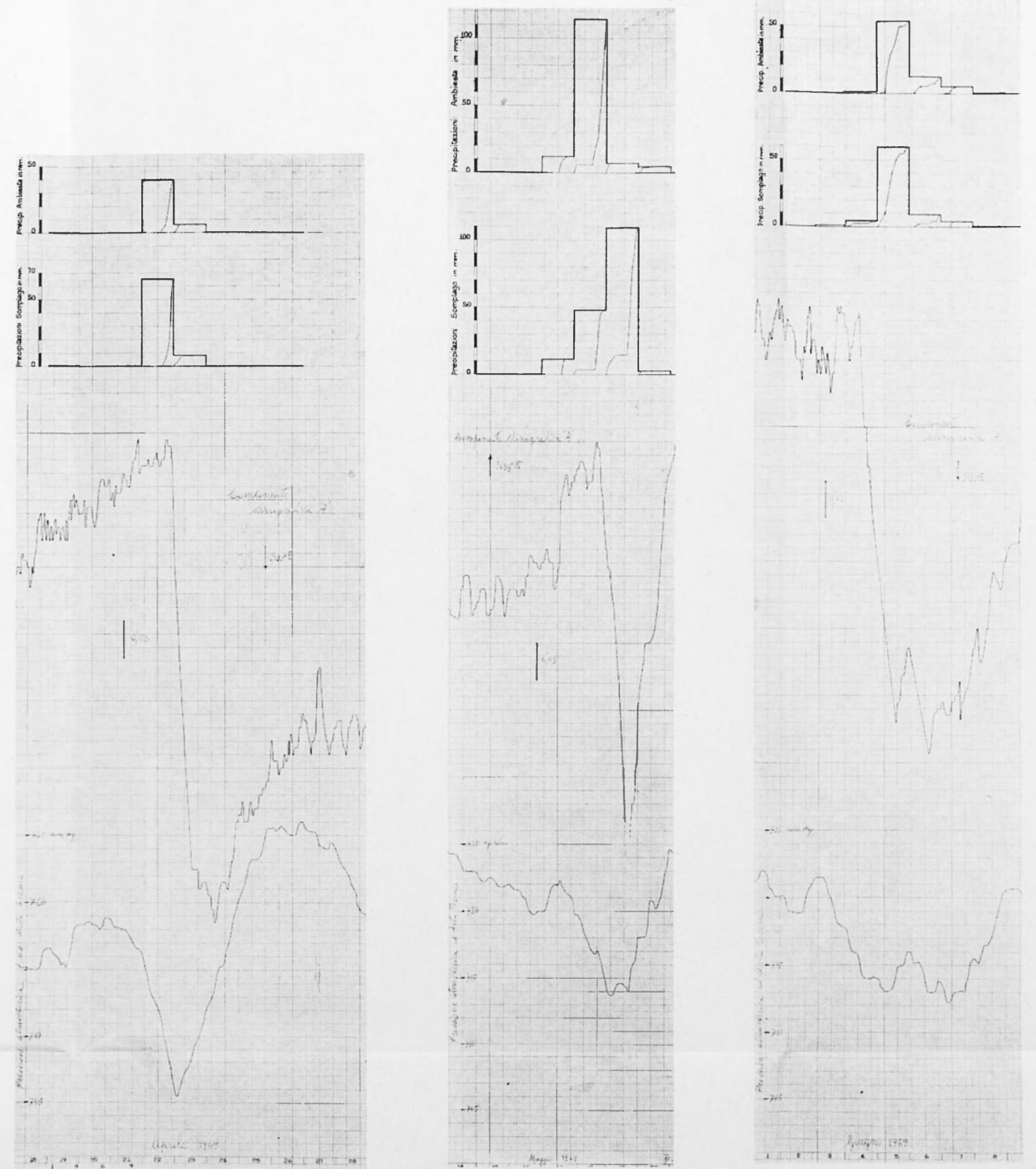

2.

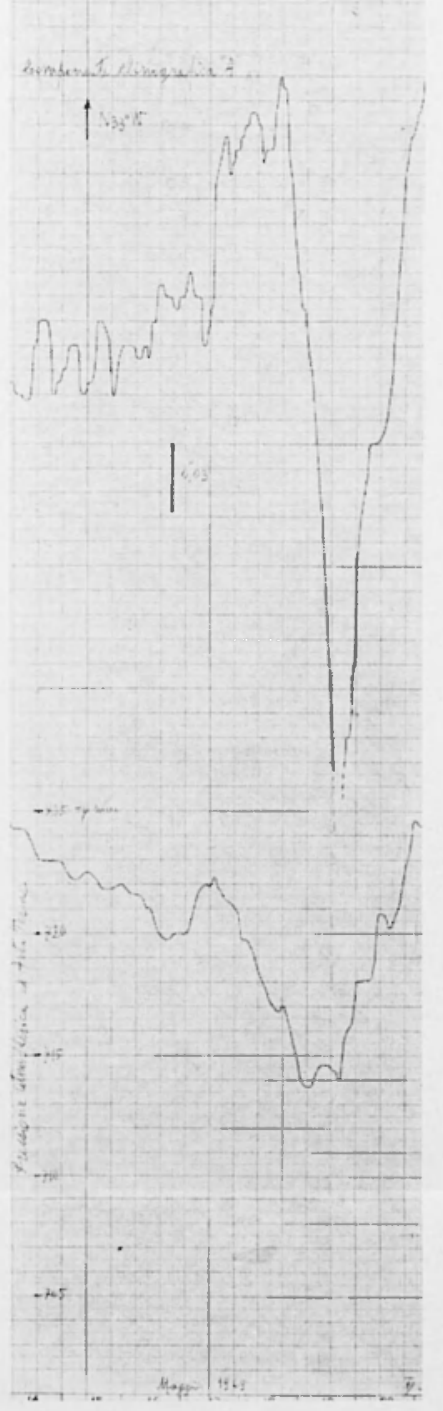

3.

Figg. 1-2-3-4-5 - Vedi figg. 6-11.

Figs. 1-2-3-4-5 - See figs. 6-11.
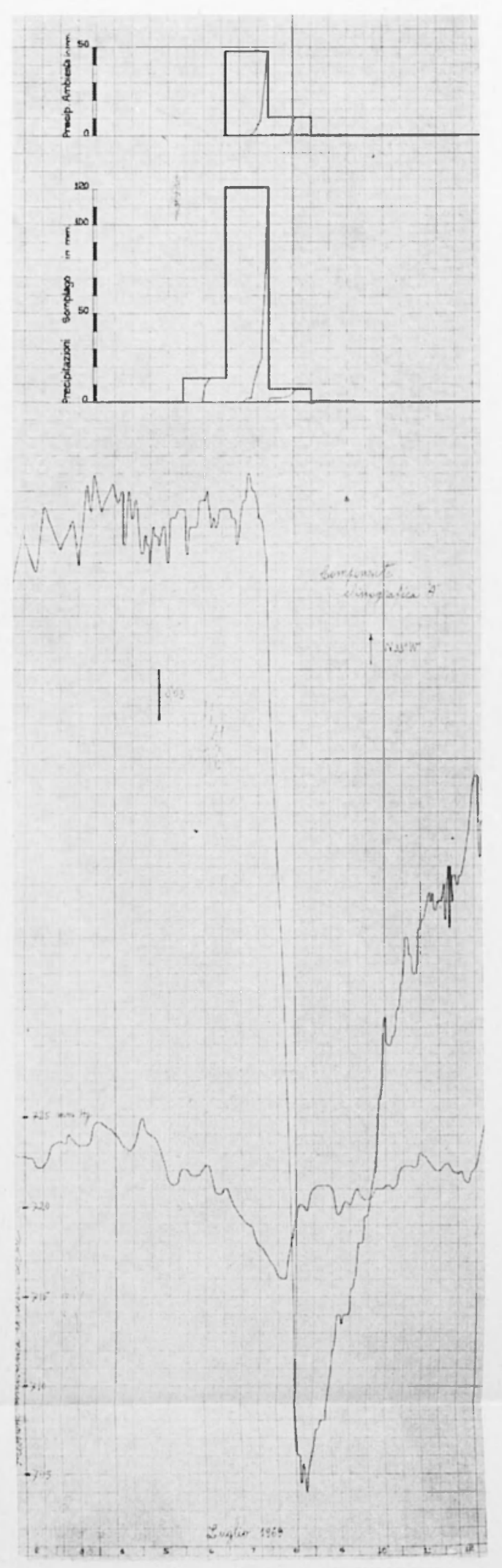

5. 

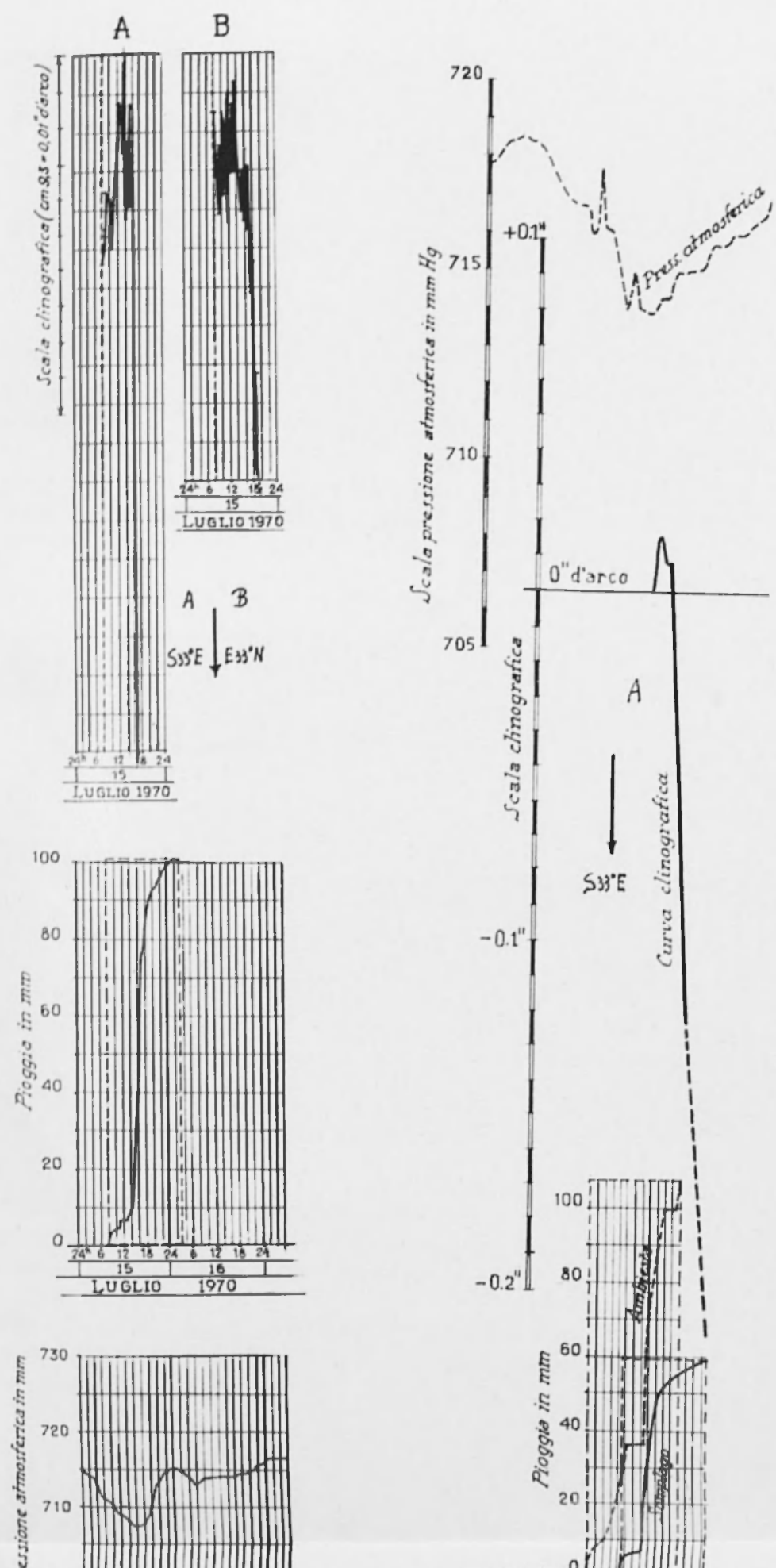

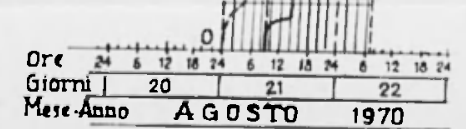
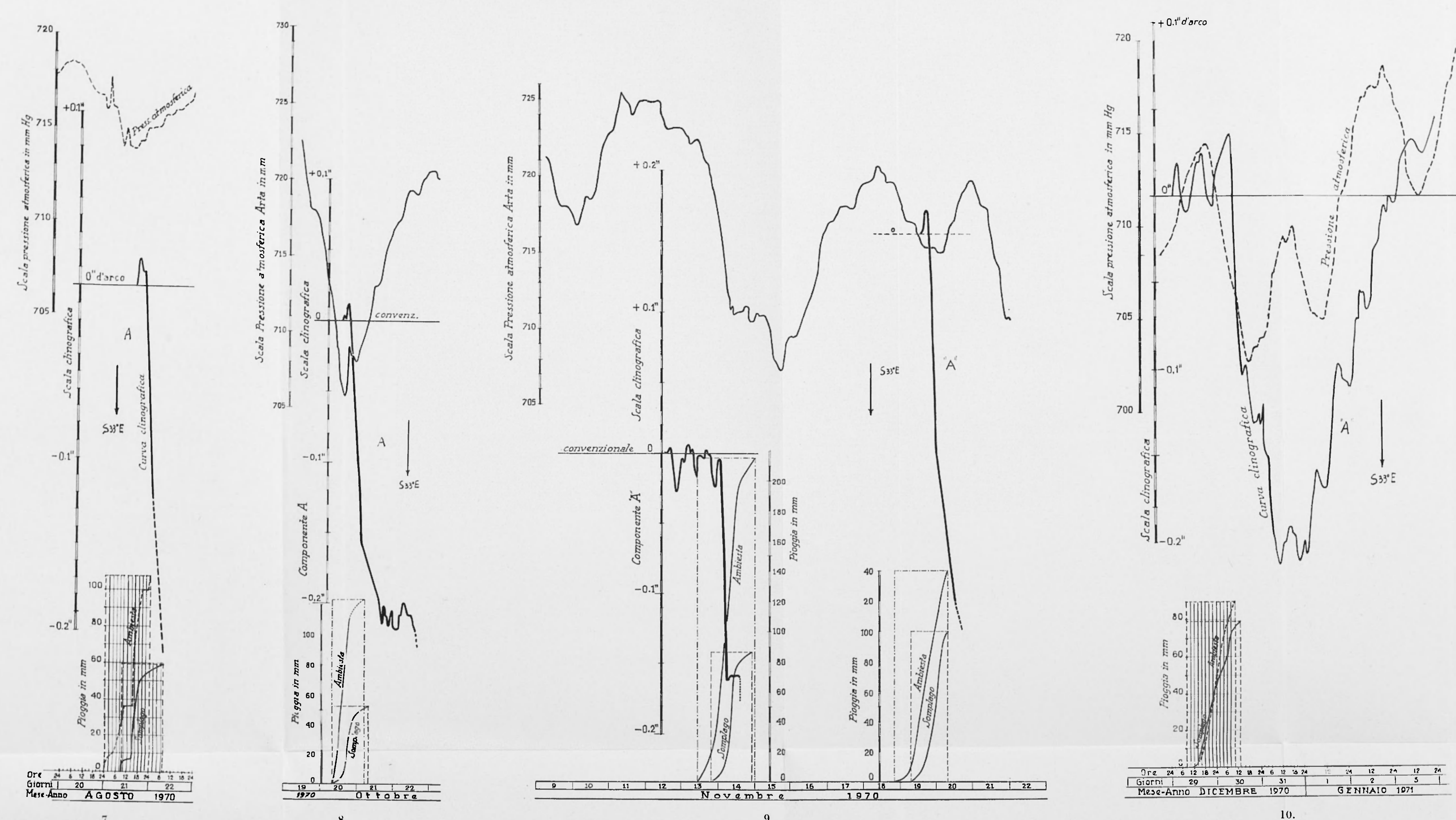

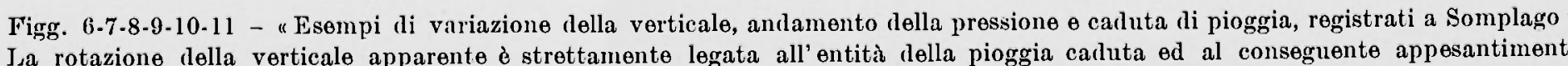

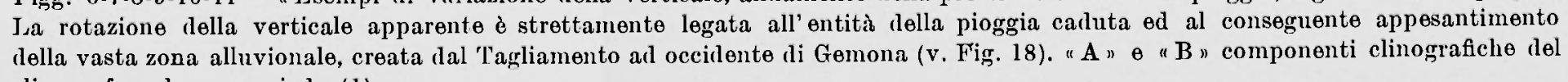
clinograto a lungono periodo (1).

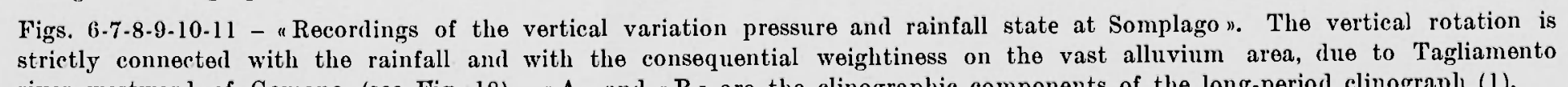

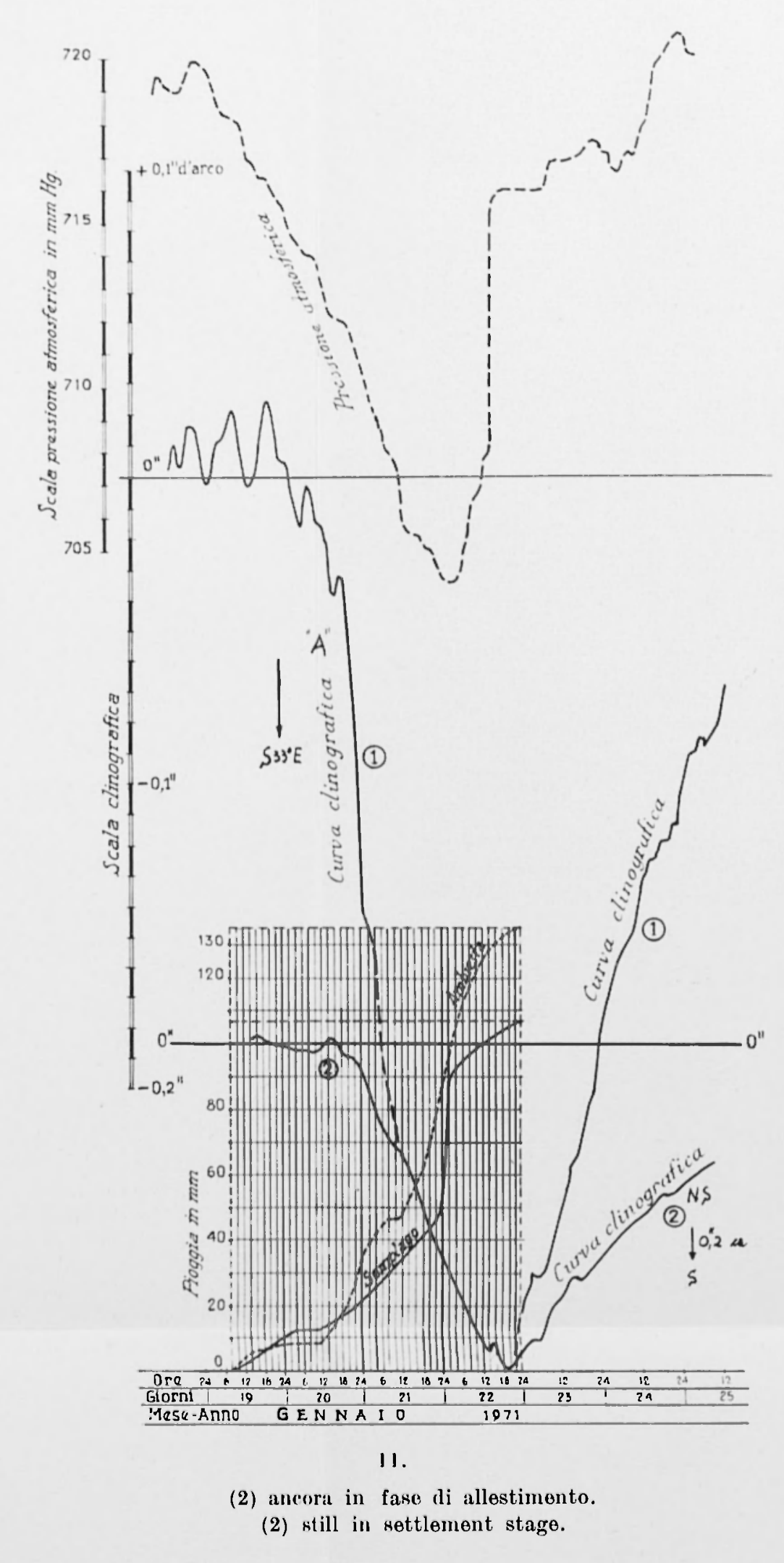



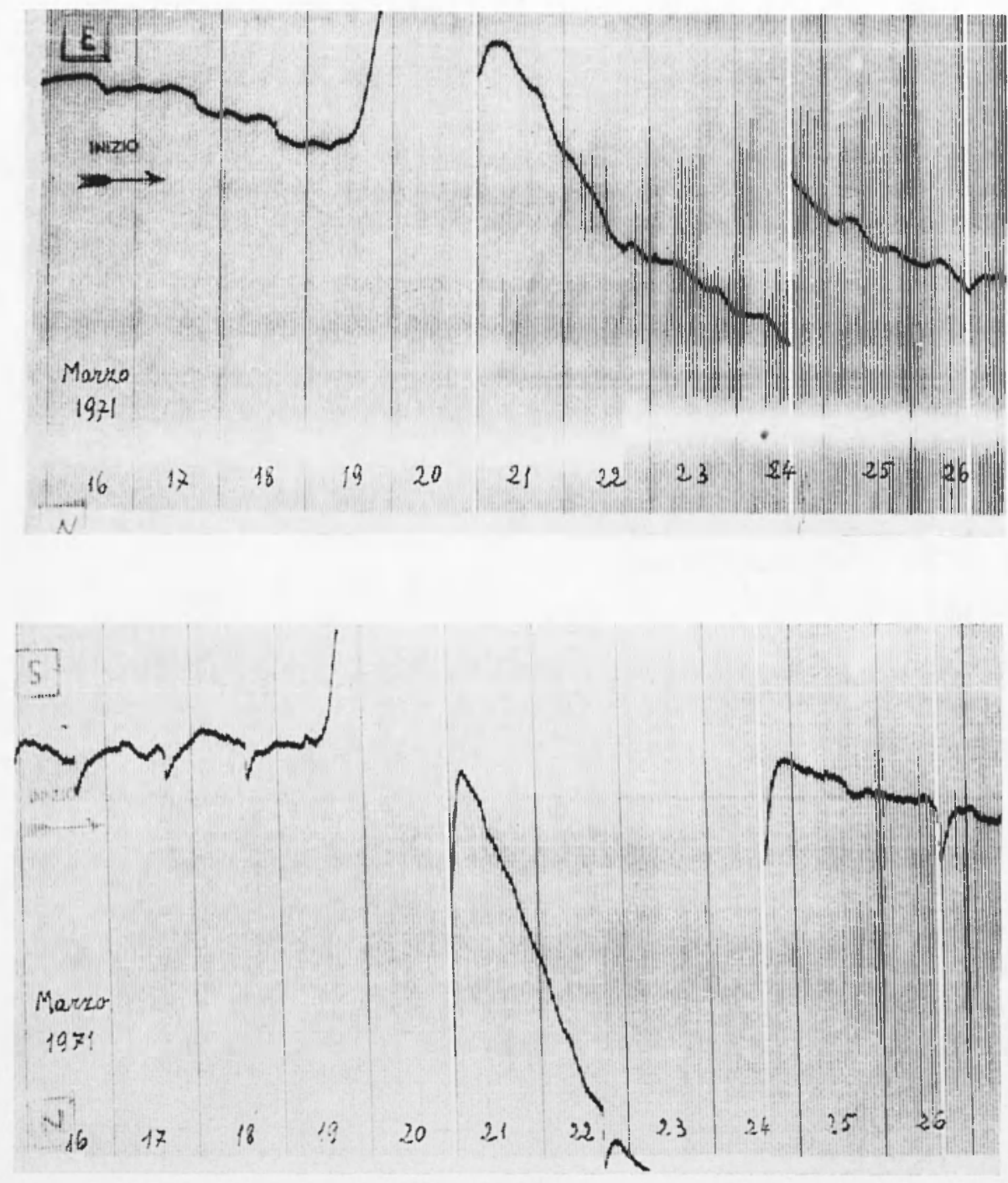

Fig. 12 - Fuoruscita amplissima di entrambe le componenti fino ad appoggiarsi ad un estremo. Impossibilità di ricostruzione del diagramma. Pioggia caduta in quantità inusitata (ca. $250 \mathrm{~mm}$ in 3 giorni), come da Fig. 13.

Fig. 12 - The amplest shifting out of recorder of both components, the image reach the end of the recorder-paper. It has been impossible to drawn the diagram. Unusual rainfall, $250 \mathrm{~mm}$ about in three days (see Fig. 13). 
5. - I movimenti clinografici, registrati a Somplago, sul margine occirlentale della faglia del lago di Cavazzo, in occasione di forti piogge, possono avere qualche legame con l'attività della faglia stessa.

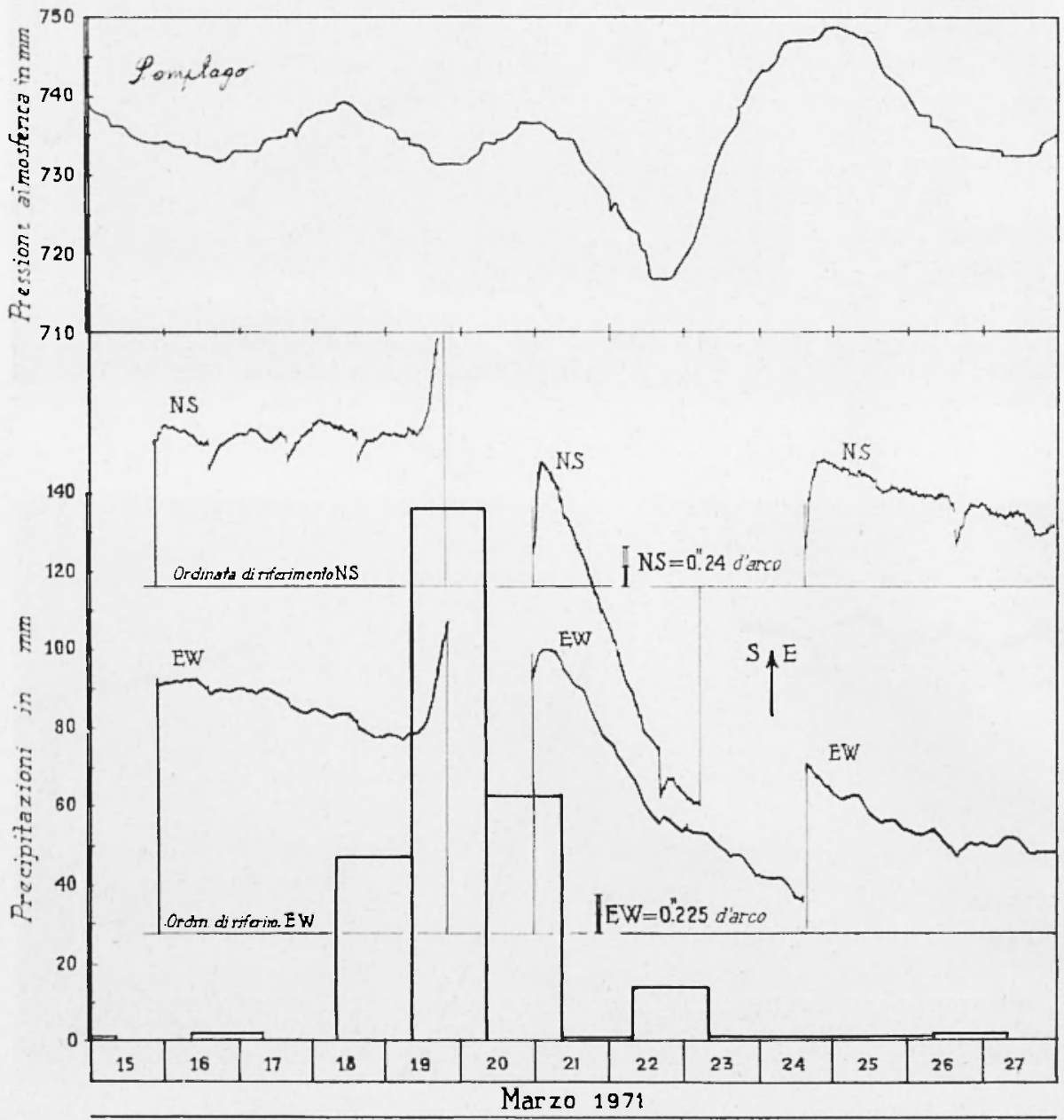

Fig. 13 - Marzo 1971: clinogramma, barogramma, pioggia.

Fig. 13 - Clinogram, barogram, rainfall of March, 1971. 

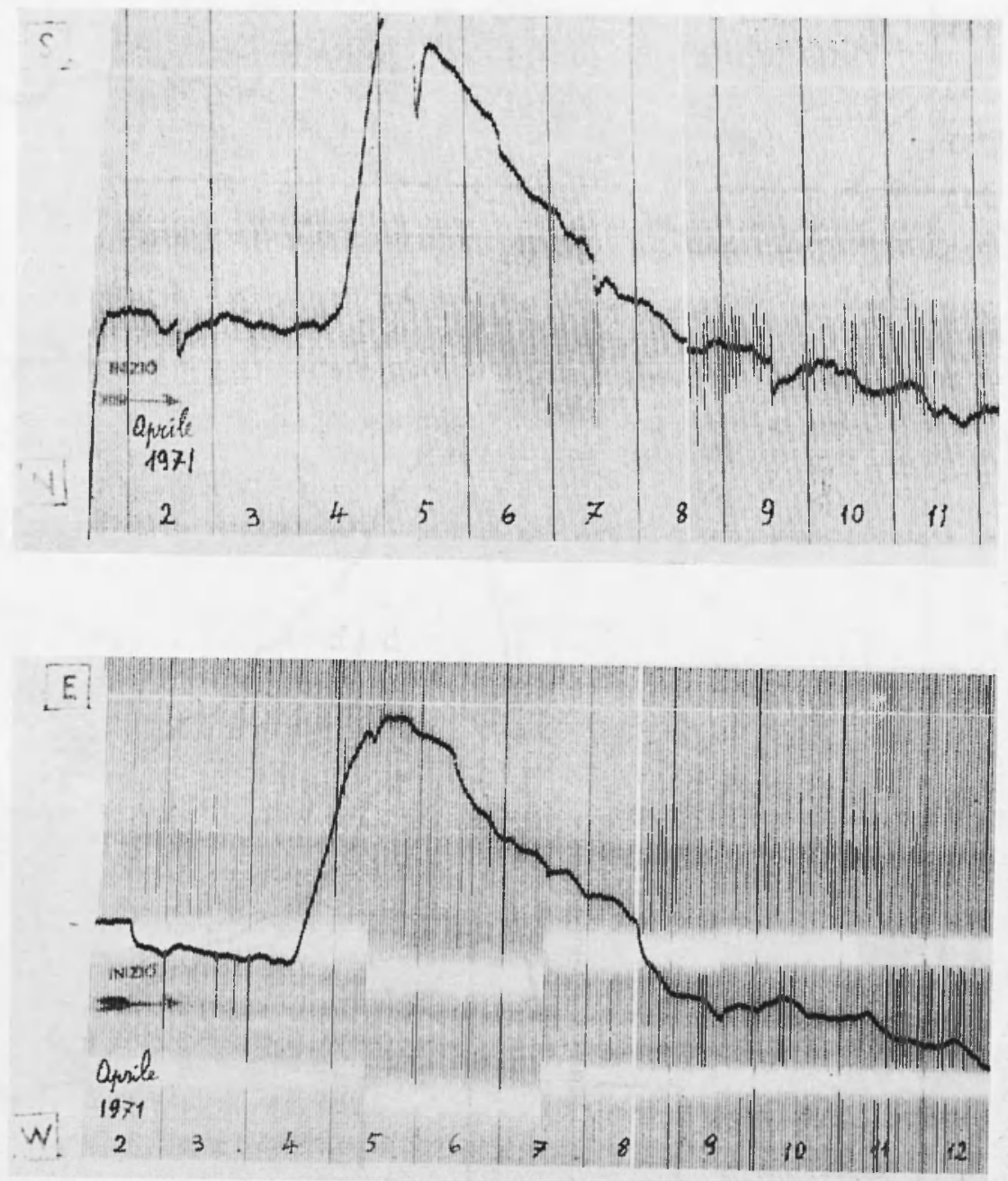

Fig. 14 - Fuoruscita della NS di aleuni cm. Deviazione provocata da pioggia (v. Fig. 15).

Fig. 14 - The NS component shifts out of recorder of some cms about. Deviation caused by rainfall (see Fig. 15). 


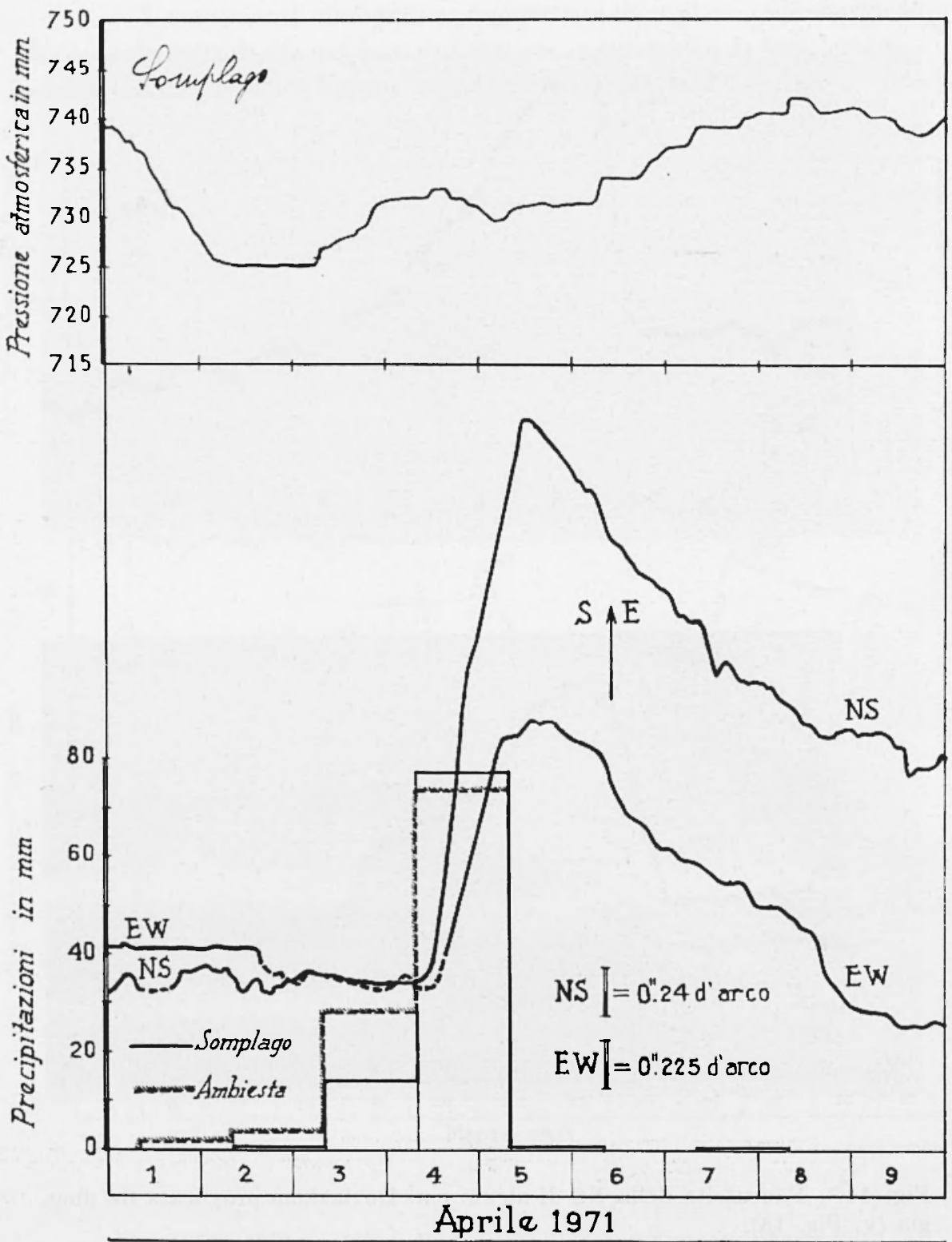

Fig. 15 - Aprile 1971: clinogramma, barogramma, pioggia.

Fig. 15 - Clinogram, barogram, rainfall of April, 1971. 


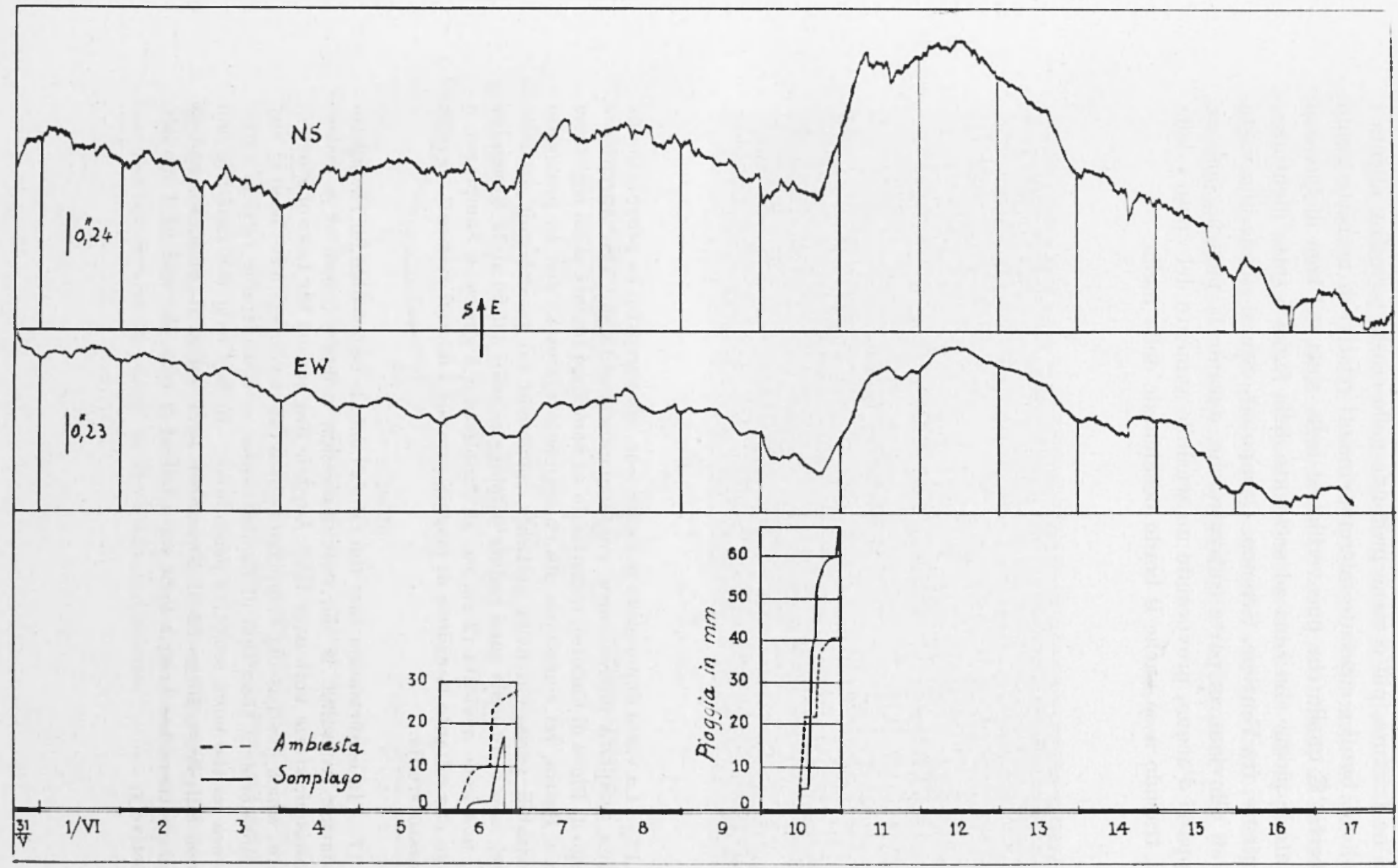

Fig. 16 - Quando la caduta d'acqua non è eccessiva il nuovo clinografo registra compiutamente le variazioni del la verticale, senza fuoruscita delle immagini, come nei due casi riportati in figura. (In corrispondenza della lieve caduta di pioggia dal 6 al 7 Gingno 1971 , la componente " $A$ " del grande pendolo ha subito uno spostamento verso $\$ 33^{\circ} \mathrm{E}$ di $\mathrm{c}$ a. $18 \mathrm{~cm}$, pari a ca. $0^{\prime \prime}, 2$ in corrispondenza del $2^{\circ}$ caso, si ebbe invece la fuoruscita dell'immagine, nello stesso verso.

Fig. 16. - When the rainfall is not heavy, the vertical variations are entirely recorded by the new clinograph: the images don't overstep the recorder-paper limits, as in two cases in figure slown. (In conformity with the scarce rainfall from 6 to 7 of June, 1971, the "A " component of great pendulum shifts of $18 \mathrm{cms}$ about (corresponding to 0 ", 2 ab.); in conformity with the second example, against, the image overstepped in the same direction the recorder-paper limits). 
Una frattura, pii o meno profonda nella crosta terrestre, allenta i vincoli dlei bordi e consente micromovimenti relativi di un certo grado di libertà. È quello che può verificarsi nella zona del lago di Cavazzo durante le piogge. Ia zona ar occidente della faglia è tutta montuosa. Arl oriente, fra Venzone, Gemona, Osoppo ed oltre, si stencle una vasta zona di alluvioni, in parte coltivate, che, durante la pioggia, possono inzupparsi d'acqua, provocando un sensibile aumento del " peso " della zona, traendo a sé anche il bordo occirlentale della faglia.

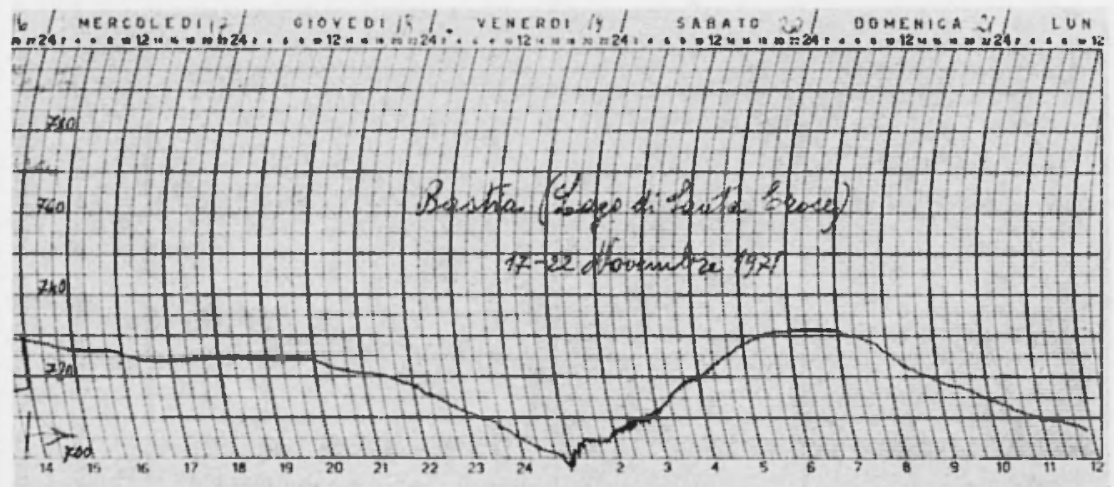

Fig. 17 - La vasta depressione in figura (che, tra parentisi, ha provocato una violenta tempesta microsismica, contemporaneamente sull'Alto Adriatico e nel lago di Pieve di Cadore), transitando su Somplago (dove è stata registrata come a Bastia, ivi comunque più chiaramente marcata) non ha provocato apprezzabili variazioni della verticale, nemmeno sui pendoli più sensibili. Difatti, non vi fu nella zona carluta di pioggia: solo, dal 20 al 22 Novembre 1971, una lieve nevicata (2 cm ca. all'A mbiesta e $1 \mathrm{~cm}$ ca. a Somplago), a riprova che soltanto la caduta di pioggia provoca i micromovimenti, oggetto di questo studio.

Fig. 17 - In confirmation that the rainfall only is responsible for the micromovements recording, is the vast depression in figure (cause of a violent microseismic storm both over High Adriatic Sea and on the Lake of Pieve di Cadore) which overpassing Somplaro (where the recordings have been in any ('ase more evident than that of Bastia) caused no considerable vertical variation even on the more sensitive pendulums. In fact over the zone the rain did not fall: from 20 to 22 of November 1971, only, at Ambiesta and at Somplago there has been a light snow-fall of $2 \mathrm{cms}$ ab. and of $1 \mathrm{~cm} \mathrm{ab}$. respectively. 
Va sottolineato il fatto che, la risultante delle flessioni osservate, è sempre diretta verso SSE, verso il baricentro della zona in questione. In tal caso però bisogna ammettere - conformemente all'ipotesi di Selli — che la faglia non si limiti al lato occidentale del Lago di Cavazzo ma prosegua verso Sud, congiungendosi alla frattura visibile fra Avasinis e il punto di confluenza del Melò nel Tagliamento (fig. 18).

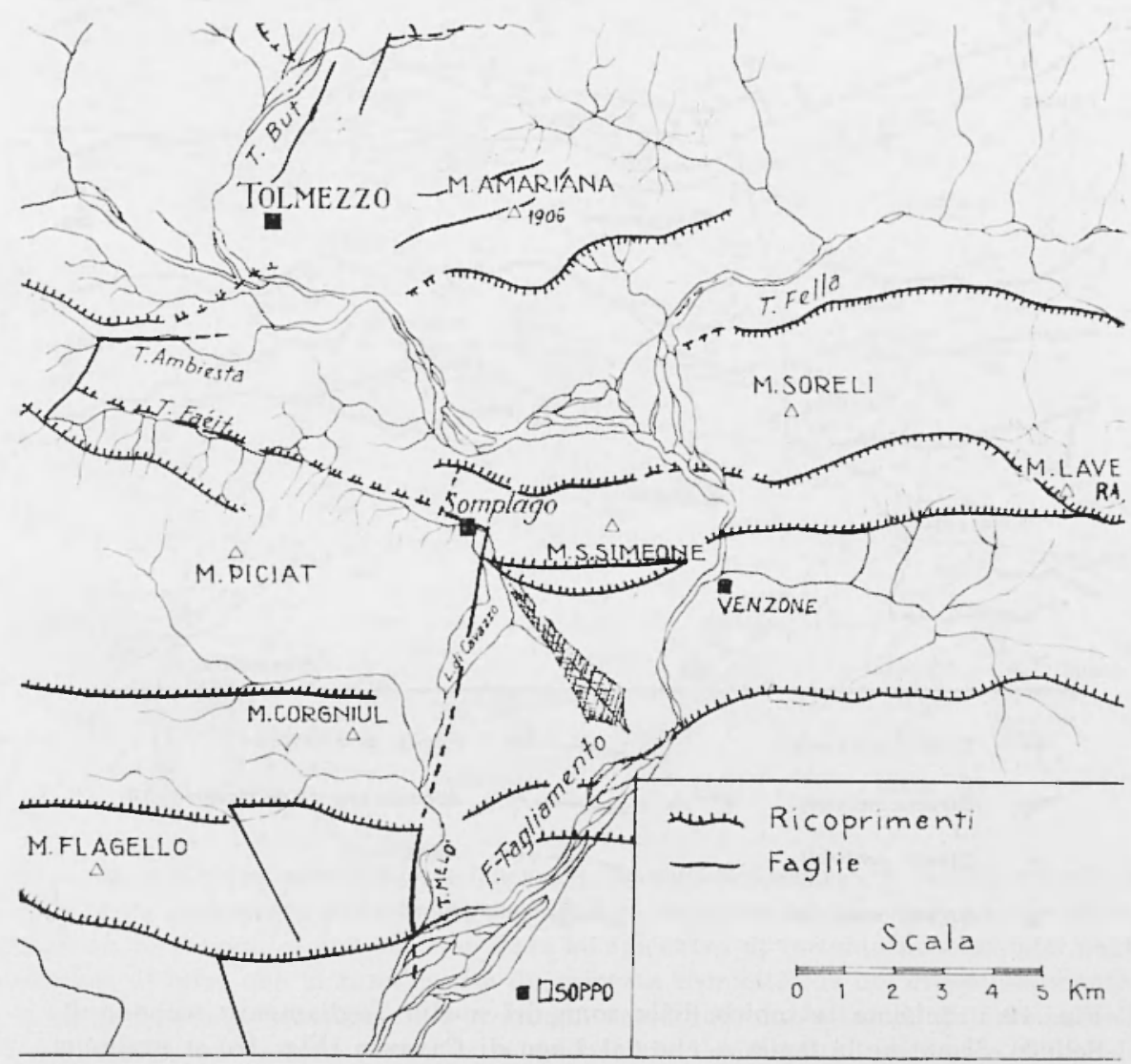

Fig. 18 - La grossa freceia in figura indica la media direzione di deviazione della verticale di Somplago, determinata dalle pioggie locali ed associata a micromovimenti della faglia del lago di Cavazzo.

Fig. 18. - The marked arrow in figure shows the mean direction of the vertical deviation of Somplago, caused by local rainfalls and associated with a micromovements of the fault of the Lake of Cavazzo. 


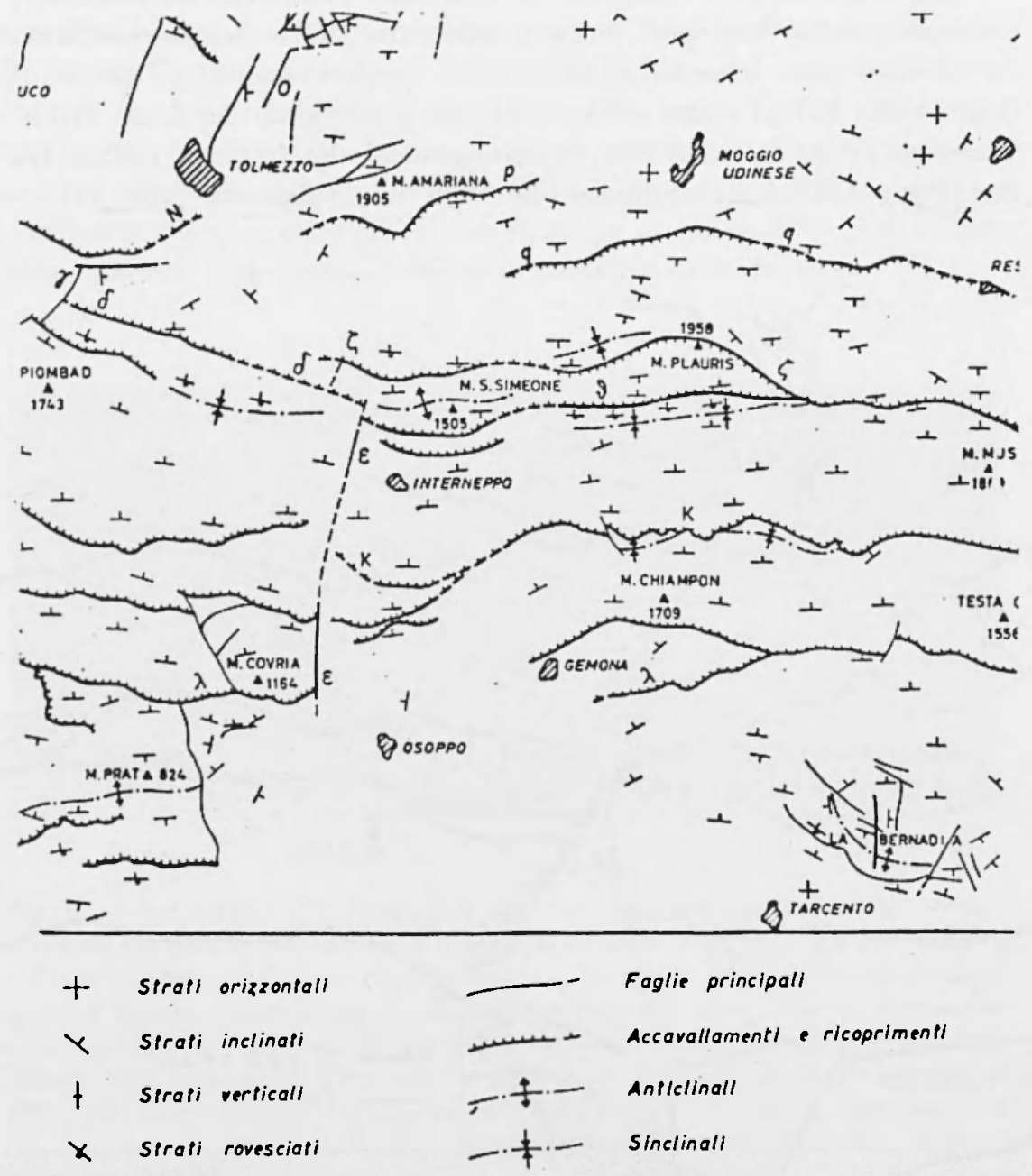

Fig. 19 - Schema tettonico della zona del medio Tagliamento secondo R. Selli $\left(^{5}\right.$ ). Si notino la faglia $\varepsilon$, che dal Lago di Cavazzo (Fig. 18) si prolunga fino nei pressi di Osoppo; nonché le faglie ad occidente del Monte Covria e ad oriente del Monte Prata.

Fig. 19 - The tectonic map of the area of the mean Tagliamento river, according to $R$. Selli $\left({ }^{5}\right)$. Worthy of remark the fault $\varepsilon$, extending from the Jake of Cavazo (Fig. 18) to environs of Osoppo and also the faults westerly of M. Covria and easterly of M. Prata. 


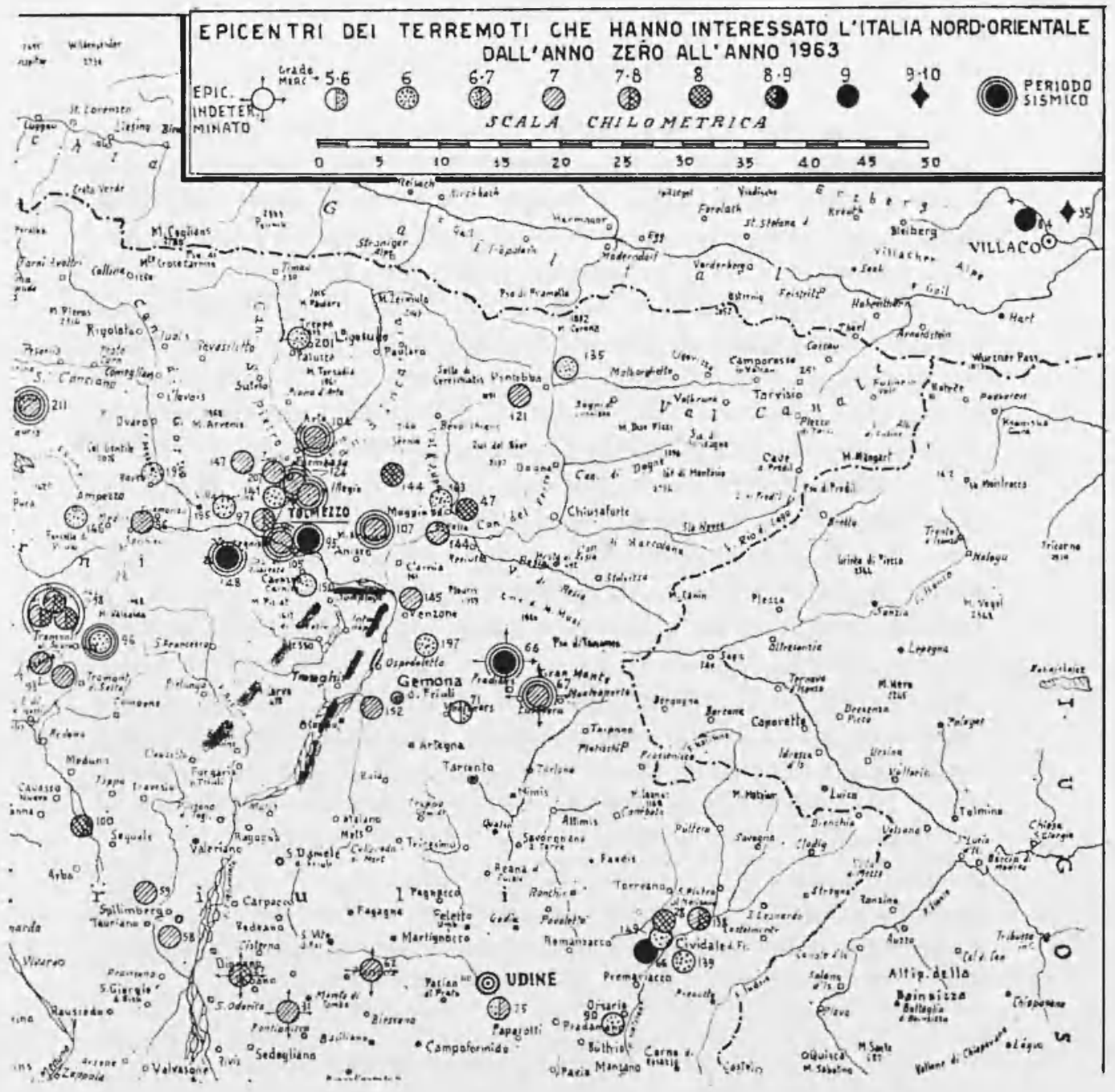

Fig. 20 - Sismicità dell'alto Vdinese, secondo Marcelli-Feliziani $\left({ }^{2} .3\right)$. Si noti come la zona del lago di Cavazzo interessata dalla faglia omonima - soggetta a micromovimenti associati alla caduta locale di piogrgia - non sia mai stata all epicentro di terremoti catastrofici negrli ultimi due millemni. Il fatto che la zona esente da spiccata sismicità (da noi grossolanamente delimi. tata in figura con largo tratteggio), si prolunghi verso Sud, fino oltre la confluenza dell'Arzino nel Tagrliamento, può essere messo in relazione con l'esistenza delle faglie ad occidente del Monte Covria e, sopra tutto, ad oriente del Monte Prata (Fig. 19), qualora queste - data la loro posizione rispetto alla valle del Tagliamento - si ritengano soggette a fenomeni analoghi a quelli riscontrati a Somplagro.

Fig. 20. - The Carnian seismicity map, according to Feliziani-Marcelli $\left({ }^{2}, \mathbf{3}\right)$. It is very interesting as the area of the Lake of Cavazzo -- with homonimous fault connected and subjected to micromovements at the local rainfall associated-never has been at the epicentre of destructive earthquakes during the last two milleniums. If the area (in figure grossly delimited by a large marks) free from high seismicity continues sonthward beyond the confluence of the Arzino in the Tagliamento River, that is in relation to existence of the faults westerly of M. Covria and, chiefly, easterly of M. Prata (Fig. 19). This one could be possible if the faults, owing to their position in respect of the Tagliamento Valley, are subjected to phenomena like the ones at Somplago found. 
Una fonrlamentale caratteristica rlel movimento è che esso si verifica con rleviazione brusca e rapirla, mentre, a pioggia ultimata, il ritorno verso la primitiva posizione d'equilibrio avviene in morlo lentissimo (figg. 15, 16); come se una fiumana sotterranea, improvvisamente alimentata, perlesse via via di portata, al cessar della pioggia.

A prescinclere dal morlo come si realizza il micromovimento della faglia rlel lago di Cavazzo in concomitanza alla carluta di pioggia (modo che potrà essere precisato in successive inclagini), resta il fatto inconsueto di una grossa frattura, animata ra una insospettata causa esterna. Se la causa è quella prospettata nel numero precerlente, non vi è rubbio che le rlue superficie a contatto della faglia presentano - quando la causa agisce - dei movimenti relativi. Ia frequenza delle pioggie, nella zona, è molto elevata: frequente, quindi, è il ripetersi di tali micromovimenti, più o meno accentuati a seconda dell'intensità della causa perturbante. Ciò consente di poter affermare che - almeno nella parte esterma, superficiale, della faglia - riesce difficile l'accumulo di grosse tensioni elastiche, continuamente "scaricate" dai micromovimenti relativi accennati. In altre parole, grazie a tali micromovimenti, nella zona non dovrebbero verificarsi terremoti superficiali rli grande intensità. Sta rli fatto che il lago di Cavazzo non si è mai trovato all'epicentro di grossi terremoti, in tempi storici. Verlasi la fig. 20, tratta da un lavoro di Marcelli-Feliziani $\left({ }^{2}, 3\right)$ : in essa, la zona del lago di Cavazzo appare circonclata ra epicentri di terremoti, anche violentissimi, verificatisi dall'anno 0 al 1963. Sembra improbabile trattarsi di pura coincirlenza.

\section{BIBI/IOGRAIIA}

(1) Crior P., 1969. - Relazioni fra sismiciti e moti lenti nella crosta terrestre. (Relations between reismicity and slow movements in the Earth's crust). "Annali di Geofisica", XXII, 3, pp. 209-228.

(2) Tilizian P., Marcent. I., 1965. - Il terremolo di Tolmezzo del 20 Aprile 19;9. I Parto: Yenti secoli di storia sismica della Regione Carnica e dell'Italia Nord.Occidentale. "Annali di Geofisica" XVIII, 3, pp. 165-207.

(3) Feldziani P., Marcel.t. I., 1966. - Il Parte: Studio macrosismico-geologieo: determinazione analitica delle grandezze ipocentrali e nalura della scossa. "Annali di Geofisica", XIX. 2, pp. 191-214.

(4) Teruglao K., 1925. - Le Prealpi fra l'Isonzo e l'Arzino. "Estr. Boll. Ass. A gr. Friulana" ".

(5) SELAI R.. 1963. - Schema geologico delle Alpi Carniche e Giulie occidentali. "Giornale di Geologia ", XXX. 